\title{
Fast-Atom Bombardment-Induced Condensation of Glycerol with Ammonium Surfactants II: Time Dependence of Mass Spectra and Tandem Mass Spectra
}

\author{
Albert A. Tuinman and Kelsey D. Cook \\ Department of Chemistry, University of Tennessee, Knoxville, Tennessee, USA
}

Studies of fast-atom bombardment (FAB)-induced condensation between trimethyltetradecylammonium cations and glycerol have been extended to consider spectral time dependence. To enhance reproducibility of time dependence, a modified FAB target was used. FAB mass spectrometry of deuterium-labeled surfactants and FAB/collision-induced dissociation (CID) of nonlabeled material demonstrate that products of condensation at the surfactant "head group" predominate early in the analysis, while tail adducts become prominent later. 'Ihis time dependence correlates with the expected surface activity of the products. It is incompatible with gas-phase reaction, but consistent with reaction in the condensed phase. Subtle variations in the surface activities of various condensation products (derived from changes in the number of hydroxyls from the reactive glycerol radical or in the position of attack along the surfactant chain) are reflected in the time dependence of FAB and CID spectra. CID spectra of deuterium-labeled cations provide evidence for intramolecular hydrogen transfer from the surfactant tail to the head within a surfactant radical. This transfer shows no significant kinetic isotope effect. (J Am Soc Mass Spectrom 1994, 5, 92-99)

\begin{abstract}
A rtifacts derived from reactions induced by the primary beam in fast-atom bombardment (FAB) and liquid secondary ion mass spectrometry (LSIMS) can complicate spectral interpretation. The range of reactions leading to artifacts is broad, and includes reduction, oxidation, substitution, addition, and Brönsted and Lewis acid-base chemistry [1]. Despite substantial progress in experimental and theoretical studies $[1-4]$, many aspects of bombardmentinduced chemistry and sampling are imperfectly understood. This results in part because the microenvironment at the site of impact is difficult to characterize; in some instances, it is unclear whether reactions take place in the gas phase, the condensed phase, or in a spatially intermediate region referred to as the selvedge, located just above the matrix surface [2]. Nevertheless, such chemistry can be indicative of the presence of certain reactive functional groups $[1,5,6]$.

Even saturated alkyl chains can undergo bombardment-induced reactions. We [7] have previously reported the FAB-induced condensation of the trimethyltetradecylammonium cation (1) with glycerol. Isomeric products 4 and 6 (Scheme I) differ only in the point of attachment of the glycerol residue to 1 and cannot be distinguished in the FAB spectrum without using labeled cations. However, these isomers are readily dif-
\end{abstract}

Address reprint requests to Dr. Albert A. Tuinman, Department of Chemistry, University of Tennessee, Knoxville, TN 37996-1600. ferentiated by tandem mass spectrometry (MS/MS) using low-energy collision-induced dissociation (CID) [7]. The most abundant CID fragment of 4 is $5(\mathrm{~m} / z$ 242), whereas CID of the "tail attached" adduct 6 gives primarily the trimethylammonium cation $7(\mathrm{~m} / \mathrm{z}$ 60 ). These assignments were verified by use of the isotopically labeled cations 2 and 3 [7]. In addition to 4 and 6, less abundant condensation product ions at masses 286 and 316 Da were observed and ascribed to the addition of glycerol fragment radicals $\left(\cdot \mathrm{CH}_{2} \mathrm{OH}\right.$ and $\cdot \mathrm{CH}(\mathrm{OH}) \mathrm{CH}_{2} \mathrm{OH}$, respectively) to surfactant radicals.

Our previous results [7] were obtained from timeaveraged spectra, that is, the accumulation of mass spectrometry data over approximately 10 minutes for each spectrum acquired. A systematic time dependence of the spectra was noted, but not considered in detail. Several studies [8-16] have previously examined parameters affecting time dependence. We now report the time dependencies of mass spectra and tandem mass spectra of these adduct-formation reactions and their implications with regard to the condensation mechanism.

\section{Experimental}

The bromide salts of 2 and 3 , synthesized [17] from the appropriately deuterated precursors as described elsewhere [18], were kindly provided by Dr. L. J. Magid. 
<smiles>CCCCC[N+](C)(C)C</smiles>

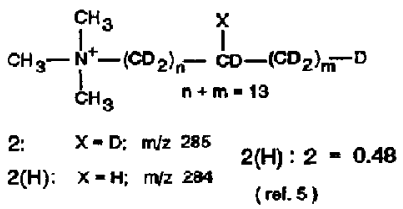

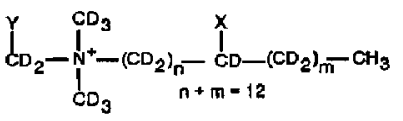

3: $\quad X=Y=D ; m / 2291$

$3(H): \quad X$ or $Y-H ; m / z 290$ $3(\mathrm{H}): 3=0.38$ (ret. 5)

Formulae<smiles>[Y][Y10](=O)O[W]</smiles><smiles></smiles>

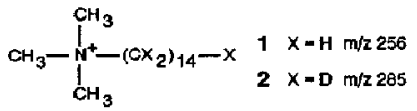

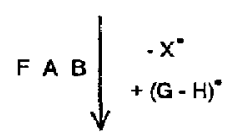
$\mathrm{CH}_{3}-\mathrm{N}_{\mathrm{n}+\mathrm{m}-13}^{\mathrm{NH}_{3}-\left(\mathrm{Cx}_{2}\right)_{\mathrm{n}}-\mathrm{l}_{3} \mathrm{CH}_{2} \mathrm{OH}}$

$6 \mathrm{X}=\mathrm{H} \mathrm{m} / \mathrm{z} 345$

$10 \quad x=0 \quad m / 2374$

Scheme I

Glycerol, $\mathrm{d}_{3}$-glycerol, and the bromide salt of 1 were obtained from Aldrich Chemical Co. (Milwaukee, WI) and used without further purification.

Mass spectra were obtained on a hybrid mass spectrometer of BEqQ geometry, the ZAB-EQ from VGAnalytical (Manchester, UK). In all cases $\sim 3 \mu \mathrm{g} / \mathrm{mg}$ solutions of the surfactant in glycerol were deposited on a modified $F A B$ target hereafter referred to as the dimple probe (Figure 1). This probe provides better
DIMPLE FAB

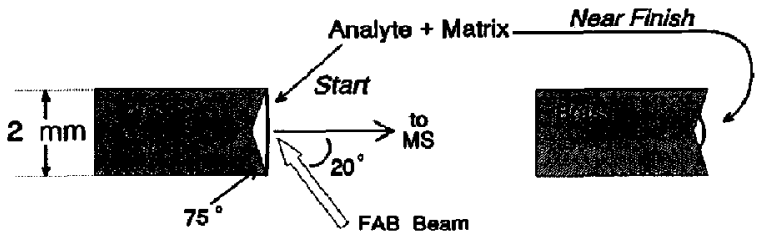

Figure 1. Cross-section of the dimple FAB probe tip used to improve reproducibility of spectral time dependencies. During bombardment, the full dimple (left) is gradually depleted, but the sample remains on axis (right).

reproducibility of the time dependencies than coating a thin film on a conventional flat probe. In particular, spectra from the dimple probe were less subject to the sudden intensity fluctuations sometimes scen with flat probes. These are thought to occur because of the generation of "islands" of matrix on the flat tip when the analysis nears completion and the probe tip is incompletely covered. Using the dimple probe, the shrinking droplet remains centered in the hollow cone, as illustrated in Figure 1.

The loaded tip was bombarded with Xe atoms generated in an Ion Tech (Middlesex, UK) saddle field source operated at $8 \mathrm{kV}$ with an emission current of 0.6 mA. Time dependencies of FAB mass spectra (Figures 2 and $3 a$ ) were established by repetitive scanning of MS-I (BE) over the appropriate mass range and subsequent reconstruction of the selected ion chromatograms.

Low-energy CID studies were done by selecting the precursor ion with MS-I ( $\mathrm{m} / \Delta \mathrm{m} \geq 1000)$, followed by decelerating the ion, collisionally activating it with $\mathrm{Ar}$ at $15 \mathrm{eV}$ in the collision quad (q), and analyzing the collision products with MS-II (Q). The Ar pressure measured with an ionization gauge outside $q$ was $2 \times 10^{-6}$ torr, at which pressure precursor peak attenuation was reduced by about $50 \%$.

Time dependencies of CID fragments were determined by one of three methods: (1) MS-I fixed at the appropriate precursor mass, and repetitive scanning of the analyzer quadrupole with subsequent manual evaluation of the relevant peak intensities (Figure 5); (2) MS-I fixed at the appropriate precursor mass, and the analyzer quadrupole jumped under computer control between the product ions of interest (Figure 3b); or (3) MS-I switched alternately (under computer control) between precursor ions of interest by changing the accelerating and electrostatic analyzer voltages, and the analyzer quadrupole jumped between product ions of interest for each chosen precursor (Figure 4).

\section{Results and Discussion}

\section{Time Dependence of Mass Spectra}

Figure 2 shows the time dependence (selected ion chromatograms) of five significant ions in the FAB 

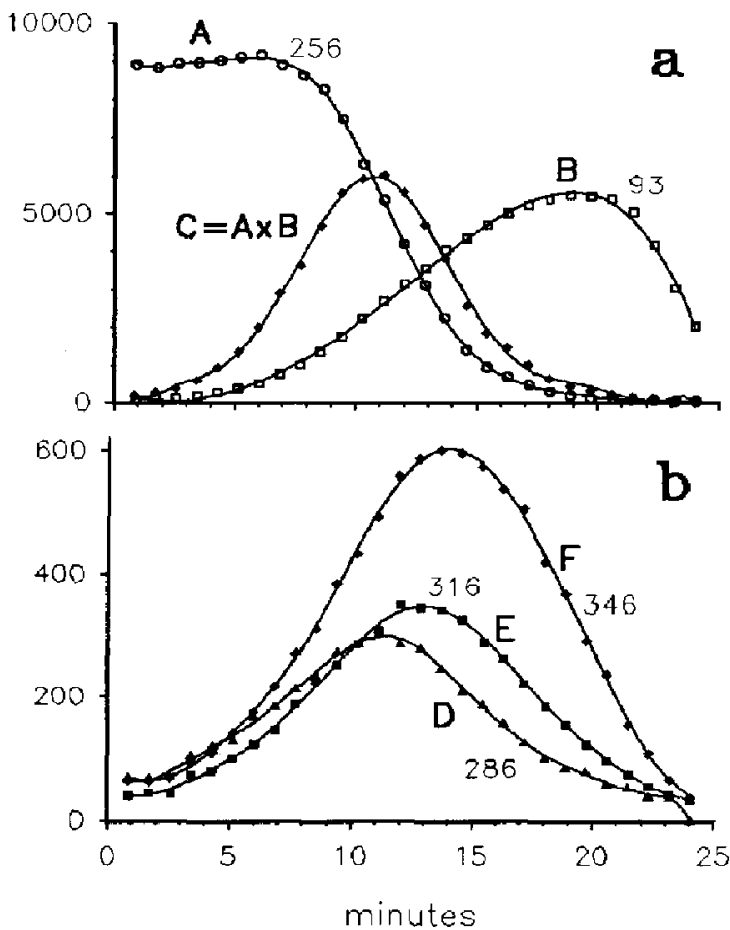

Figure 2. Time-dependent intensities at the indicated mass-tocharge ratios for $F A B$ of $\mathbf{1}$ in glycerol (Scheme I). Trace $\mathbf{C}$ is the algebraic product of traces $A$ and $B$, multiplied by an arbitrary factor to bring it on scale. Solid lines in this and subsequent figures are arbitrary fits to facilitate comparison.

mass spectrum of a solution of the unlabeled surfactant 1 in glycerol. Included are $m / z 256$ (the surfactant cation, trace $A$ ), $m / z 93$ (protonated glycerol, trace B), and the adducts with $m / z 286,316$, and 346, traces $D$, $E$, and $F$, respectively. (Trace $C$ is the algebraic product $A \times B$, see below.) At first, the strongly surface active cation 1 suppresses ions from glycerol almost completely $\left(\mathrm{I}_{256} / \mathrm{I}_{93}>100\right.$, where $\mathrm{I}_{m / z}$ denotes the intensity at the indicated mass-to-charge ratio). As the signal for 1 decreases, those for the matrix (represented by $m / z$ 93) increase through a maximum, then decrease as the sample evaporates to dryness. Such behavior is normal for surfactant solutions in glycerol $[11,13-16]$. An extraurdinary ubservation, huwever, is that the abundances of the three adducts (Figure 2b) maximize near the point in time when the glycerol and surfactant ion abundances are equal (Figure 2d). Although initial surfactant concentration does affect the times and intensities of the chromatogram maxima, the general appearance of the chromatograms is similar for all concentrations examined (2-10 $\mathrm{mM}$ ), that is, adduct peaks are always most intense near the point where surfactant and protonated glycerol abundances are equal. The abundance maxima for adducts always appear in the sequence $m / z 286,316,346$.
Similar bombardment-induced chemistry in FAB has variously been attributed to condensed-phase (solution or selvedge) or gas-phase chemistry [2]. As will be shown, a case can be made for either alternative if arguments are based solely on the time dependence of FAB spectra of unlabeled material. In contrast, the time dependence of FAB spectra of selectively labeled 2 , and of CID spectra of the nonlabeled 1 will be shown to provide unambiguous evidence of sampling of adducts from the condensed phase.

\section{Predictions Assuming Gas-Phase Reaction}

FAB with 8-keV xenon atoms is expected to generate a cloud of sputtered species that, because of the high energy of the incident atoms, contains a wide array of bombardment-damaged products (including glycerol radicals [3] and surfactant radical cations) as well as neutral and protonated glycerol and undamaged surfactant cations. Adduct formation might then proceed via a bimolecular gas-phase reaction, so that $I_{A} \alpha$ $\left[(G-H)^{-}\right] \times\left[(1-H)^{+\cdot}\right]$, where $I_{A}$ is the observed abundance of the adduct ion, and $[(G-H)]$ and $\left[\left(1-H^{+\cdot}\right]\right.$ are the gas-phase concentrations of the radicals formed by hydrogen abstraction from glycerol and surfactant, respectively. Although it is not possible to measure $\left[(\mathrm{G}-\mathrm{H})^{*}\right]$ and $\left[(1-\mathrm{H})^{+\cdot}\right]$ directly, it seems reasonable to assume that they would be proportional to the intensities of protonated glycerol and the surfactant cation, respectively (i.e., traces B and A of Figure 2). The time dependence of $I_{A}$ expected for gas-phase reaction can therefore be estimated by calculating the algebraic product of the observed intensities of $(\mathbf{G}+\mathbf{H})^{+}$and 1 , as depicted in trace $C$ of Figure $2 a$. Although trace $C$ does not coincide exactly with $D, E$. or $\mathrm{F}$, the qualitative agreement is reasonable. The gasphase reaction mechanism certainly cannot be excluded on the basis of this experiment.

\section{Predictions Assuming Condensed-Phase Reaction}

In an alternative mechanism, $(\mathrm{G}-\mathrm{H})^{\circ}$ and $(\mathbf{1}-\mathrm{H})^{+\cdot}$ formed by energy transfer from the impinging $\mathrm{FAB}$ beam could combine to form products (i.e., 4 and 6 ) in the condensed phase. The products would be formed (presumably rapidly) from the onset of bombardment, but not necessarily sampled immediately.* Kinetics would be complex, but if sampling was selective for certain products, the remaining products would accumulate in solution. The observed time dependence of product ion abundances (Figure $2 b$ ) would reflect the rates at which products are sampled (reflecting competition for surface sites [13-15]), as well as the rates at which those products are formed.

\footnotetext{
* Alternatively, adducts formed from radicals in the gas phase or selvedge could be redissolved in solution [3]. In such an instance, the gas-phase surfactant radicals would have to be in an electrically neutral form (e.g., as the bromide salt) to avoid immediate acceleration into the mass spectrometer.
} 
Two factors need to be considered to predict the time dependence in the case of condensed-phase reaction. First, as noted in the discussion of Figure $2 \mathrm{a}$, it is common for more surface-active components to be sampled preferentially to those of lesser surface activity [13-15]. Second, the surface activity of materials containing a hydrophilic "head-group" (e.g., the trimethylammonium group of surfactant 1) and a hydrophobic "tail" (e.g., the $C_{14}$ saturated chain of 1) will be significantly diminished if a hydrophilic group is attached to the tail [19]. Thus, the products of condensation of 1 with glycerol (e.g., 6, which contains three hydrophilic hydroxy groups) should be less surface active than 1 itself. If both 1 and 6 are present in solution, sampling of 6 will be suppressed until the concentration of $\mathbf{1}$ is significantly diminished by the desorption process. The time dependence reflected by Figure 2 is therefore qualitatively compatible with reaction in the condensed phase.

\section{Evidence for Condensed-Phase Versus Gas-Phase Reaction}

Because the FAB time dependence (Figure 2) is compatible with either the gas-phase or condensed-phase mechanisms (as described above) a decper level of analysis is required to distinguish between these mechanisms. This proved feasible by differentiating between the products of condensation at the head and tail of the surfactant. As discussed previously (Scheme I), these products can be distinguished either by FABMS of a surfactant selectively labeled with deuterium, or by FAB-MS/MS of nonlabeled material [7]. Figure $3 a$ presents the time dependence of key FAB signals for products of head attack (8, Scheme $\mathrm{I} ; \mathrm{m} / z$ 375) and tail attack (10, Scheme $\mathrm{I} ; m / z$ 374) during the course of FAB analysis of the selectively labeled surfactant 2 . Similarly, the time-dependent abundances of CID fragments 5 (derived from the head-attack precursor 4) and 7 (derived from the tail-attack precursor 6) from the unlabeled adduct are plotted in Figure $3 \mathrm{~b}$.

Figure $3 a$ and $b$ show a very significant time dependence of the ratio of products of head and tail attack. This constitutes unequivocal evidence for sampling from (and by implication, reaction in) the condensed phase, as follows. If the reaction occurred in the gas phase, the condensation products would be sampled immediately upon formation, because they are charged and subject to the effects of the accelerating voltage, without the inhibiting effect of the viscous (glycerol) matrix. The formation of surfactant and glycerol radicals would be concurrent with the vaporization process, and there is no reason to expect that the ratio of head/tail attack (i.e., the ratio of surfactant head radicals to surfactant tail radicals) would vary as a function of time. The expected time dependence for each of the products (head and tail attack) would be similar to trace $\mathrm{C}$ of Figure 1a. The abundances would differ for

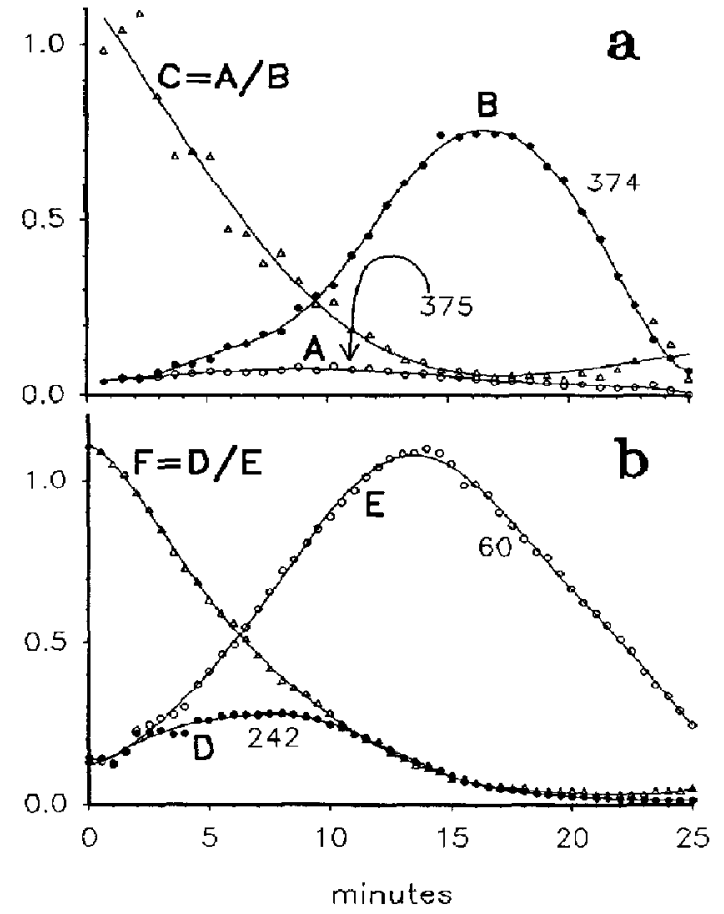

Figure 3. Time-dependent intensities at the indicated mass-tocharge ratios. (a) Products generated in situ by FAB of 2 in glycerol (Scheme I). Raw data have been corrected for contributions from mislabeled material [2(H) and ${ }^{13} \mathrm{C}$-content; see text]. (b) CID fragments from a precursor at $m / z 346$ generated in situ by FAB of 1 in glycerol (Scheme I).

each trace, but the ratio of the two product abundances would remain constant as a function of time. Traces $C$ and $F$ of Figure 3 are not readily compatible with this gas-phase-reaction hypothesis.

On the other hand, if adducts accumulate in the condensed phase, they would be sampled during FAB analysis in the order of their surface activities, reflecting competition for surface sites with each other and with the other components in the condensed phase. Head attack (addition of a hydrophilic group onto the hydrophilic head of the original surfactant) will perturb the surface activity little, if at all [19]; the surface activity of 4 and 8 should resemble that of the precursors 1 and 2. In stark contrast, the addition of a hydrophilic group onto the hydrophobic tail will significantly reduce the surface activity [19]. Because of their similar surface activities, the head-attack product and the unreacted surfactant should be sampled with similar efficiency in the FAB experiment. The less-surfaceactive tail-attack product(s) will be sampled less efficiently and accumulate in the matrix until reagent depletion slows the reaction, and the other components become substantially depleted by desorption. This is precisely the observation made in Figure 3, which therefore bolsters the condensed-phase-reaction hypothesis. 
The apparent anomalous increase in the ratio of head- to tail-attack products near the end of the analysis (Figure 3 , curve $C$ ) results from the increasing contributions of the "peak at every mass" background, which predominates after prolonged $\mathrm{FAB}$ of glycerol solutions $[3,20]$. The selectivity of CID diminishes this effect, so that Figure $3 b$ better reflects the time dependence, at least in the latter stages of the analysis. Even so, it should be noted that the observed abundance ratio of CID fragments 5 and $7(\mathrm{~m} / z 242$ and 60 in Figure $3 b$ ) does not necessarily reflect the absolute abundance ratio of the precursor ions 4 and 6 , because the corresponding reaction cross sections (for $4 \rightarrow 5$ and $6 \rightarrow 7$ ) are probably different. Because CID conditions do not change during the course of the analysis, however, those cross-sections remain constant, and the observed changes in the signal intensity ratios reflect real changes in the precursor ion concentrations.

Accumulation of reaction products in the condensed phase followed by sampling in the order of surface activity of the products also accounts for the slightly different time dependencies (Figure $2 b$ ) of the adducts. The adducts with $m / z 286$ have only one hydrophilic hydroxyl group in the glycerol residue attached to the surfactant. When attached to the tail, this residue should introduce less hydrophilic character, in comparison with the analogous product of $m / z$ 346, which has three hydroxy groups. Less hydrophilic perturbation of the tail means greater surface activity and earlier sampling. Thus, as noted earlier, the abundance of $m / z 286$ peaks before that of $m / z \quad 316$, which precedes that of $m / z$ 346. The onsets of the three curves in Figure $2 b$ are roughly coincident, representing contributions from the head-attached adducts, all of similar surface activity.

\section{Evidence for Variation in the Position of Tail Aftachment}

The discussion above contrasts the surface activity of the products of head and tail attack. There are, however, 14 potential sites of tail attack, each of which would give rise to a product of distinct surface activity. Structure 10 therefore may represent a mixture of isomeric adducts. The generation of a product mixture can be verified by the time dependence of appropriate CID spectra. Figure 4a shows the time-dependent abundances of the CID fragments 7 and 11 (precursor 10; Scheme I) in traces A and B, respectively (trace $C$, for $m / z 62$, will be discussed below). These traces qualitatively reflect the time dependence of the adduct abundance (analogous to trace $E$ of Figure $3 b$ ). There is, however, a systematic difference between traces $4 \mathrm{~A}$ and $4 \mathrm{~B}$ as reflected in the continuously changing abundance ratio (trace $4 \mathrm{D}$ ). This time dependence can be explained using Scheme II. The CID fragments at $\mathrm{m} / \mathrm{z}$ 60 (7) and 61 (11) stem from competitive transfer of $\mathrm{H}$ (from the glyceryl moiety) or D (from the labeled surfactant chain), respectively. It is reasonable to as-
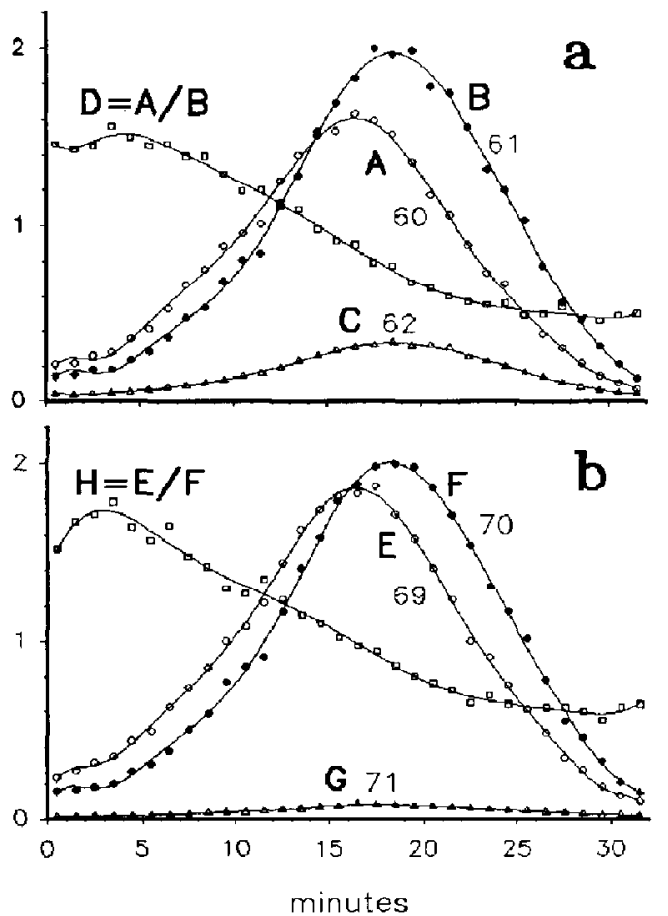

Figure 4. Time-dependent abundances of the CID fragment ions at the indicated mass-to-charge ratios. (a) Precursor ion is $m / z$ 374 , generated in situ by FAB of 2 in glycerol (Scheme IV). (b) Precursor ion is $m / z$ 380, generated in situ by FAB of 3 in glycerol (Scheme IV). Data sets were recorded simultaneously from an equimolar mixture (see Experimental).

sume that $\mathrm{H}$-transfer will he less favorable (versus D-transfer) for larger values of $n$ (i.e., when the $\mathrm{H}$ bearing glyceryl moiety is further from the ammonium group). In the absence of a significant kinetic isotope effect (see below), the diminishing relative abundance of CID fragment 7 (at $m / z 60$ ) is therefore attributed to a continuous decrease in the relative contribution from adducts with smaller $\mathrm{n}$ values. This follows not from a change in the rate of formation of the various adducts, but from differences in the sampling efficiencies of the various isomers of 10 (i.e., different values of $\mathrm{n}$ ). As expected, an analogous relationship is

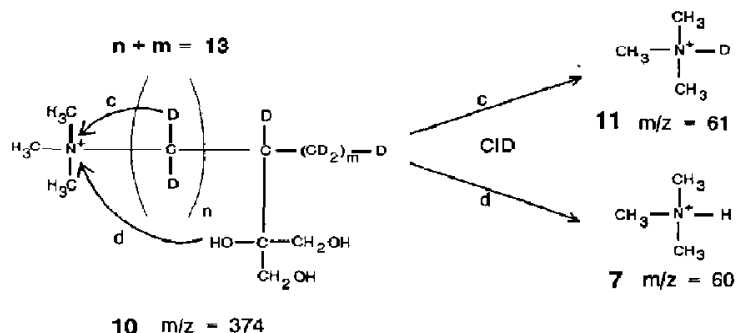

Scheme II 
seen in Figure $4 \mathrm{~b}$ for the fragments $(\mathrm{m} / \mathrm{z} 69$ and 70$)$ resulting from CID of the precursor ion at $m / z 380$, which is derived from condensation of glycerol with 3 . The labeling of 3 differs from that of 2 , and the results from 3 are useful in assessing intramolecular hydrogen transfer (described below).

The observed change in the CID abundance ratios $I_{60} / I_{61}$ and $I_{69} / I_{70}$ is consistent with the expected surface activity of the constituents of the tail-attack adduct 10. The surface activity of the starting surfactant 2 is decreased by any bonding of a hydrophilic group onto its aliphatic tail, and the magnitude of the perturbation will be greater for bonding farther away from the cationic head group [19]. Because competitive FAB desorption favors cations of higher surface activity, it is reasonable for those constituents of $\mathbf{1 0}$ with small values of $n$ (and thus a greater likelihood of $\mathrm{H}$ transfer) to predominate at the start of the analysis. It is not possible from these data to discern contributions from individual adducts of a given $n$, but the smooth trends evident in traces $\mathrm{D}$ and $\mathrm{H}$ of Figure 4 suggest a gradual and continuous shift toward increasing $n$. The marked time dependence evident in Figure 4 shows that even subtle differences in surface activities among constituents of a mixture can affect observed signal intensities, and highlights the danger of using such intensities as a quantitative analytical probe without taking possible time dependence into account.

\section{Intramolecular Hydrogen Transfer in the Formation of Tail Adducts}

Two mechanisms for formation of tail-adduct products from the differentially labeled 2 are depicted in Scheme III. Route a proceeds by abstraction of a deuterium atom from the chain with subsequent attachment of a

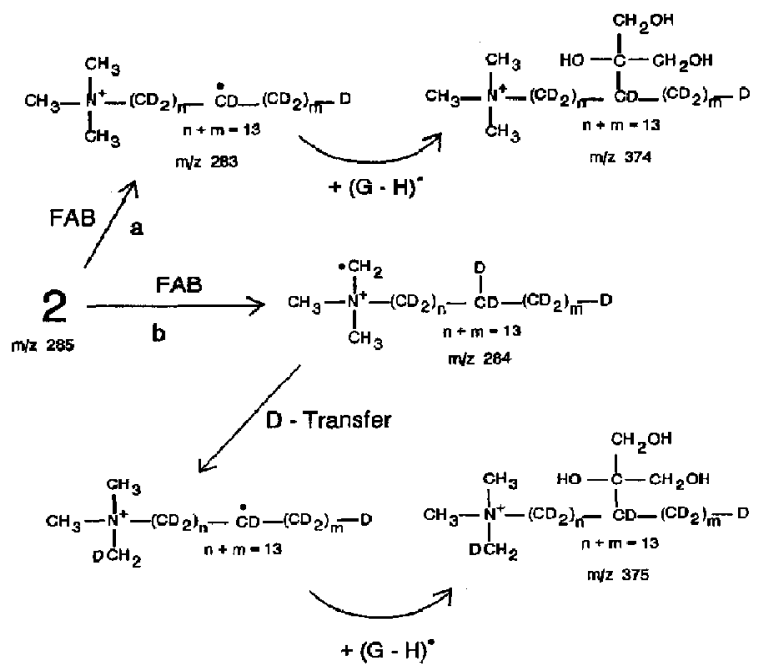

Scheme III glycerol radical onto the chain. Route $b$ involves $a b-$ straction of a hydrogen from the head group, followed by intramolecular transfer of a deuterium atom from tail to head, and attachment of glycerol to the tail. The greater stability of a secondary radical localized away from the cationic site, relative to a primary radical $\alpha$ to that site, predicts that this intramolecular deuterium transfer is energetically feasible [21-23]. However, the contributions from routes $\mathbf{a}$ and $\mathbf{b}$ of Scheme III cannot be assessed directly from the relative intensities of ions at $m / z \quad 374$ and 375 because the latter has contributions from head attack (see 8; Scheme I). Furthermore, the known isotopic impurity $\mathbf{2}(\mathbf{H})(\mathbf{2}(\mathbf{H})$ represents mislabeled 2, containing one deuterium ton few; it comprises $32 \%$ of the labeled surfactant [18]) can generate the tail adduct 12 with $m / z \quad 374$ via route b (Scheme IV; the deuteriums displayed on alkyl chains in Scheme IV are representative of the perdeuteration of those chains). This complication actually provides a means of assessing the contribution of route $b$, using CID. Whereas $C I D$ of 10 will produce fragments 7 and 11 (Scheme II; also reproduced for comparison at the left of Scheme IV), CID of 12 will produce 13 and 14 (Scheme IV). The contribution of route $b$ can thus be estimated from the relative intensities of the CID product signals from the isomeric precursors 10 and 12, provided that the observed abundance at $m / z 61$ (Figure $4 \mathrm{a}$, trace B) can be deconvoluted into the components arising from 11 and its isomer 13 (Scheme IV). The deconvolution requires knowledge of the relative reaction cross-sections for the competing fragmentations, that is, hydrogen migration from the glyceryl moiety and deuterium migration from the hydrocarbon chain. This knowledge can be obtained from the analogous reactions of 3 (Scheme IV). Tail-adduct for-

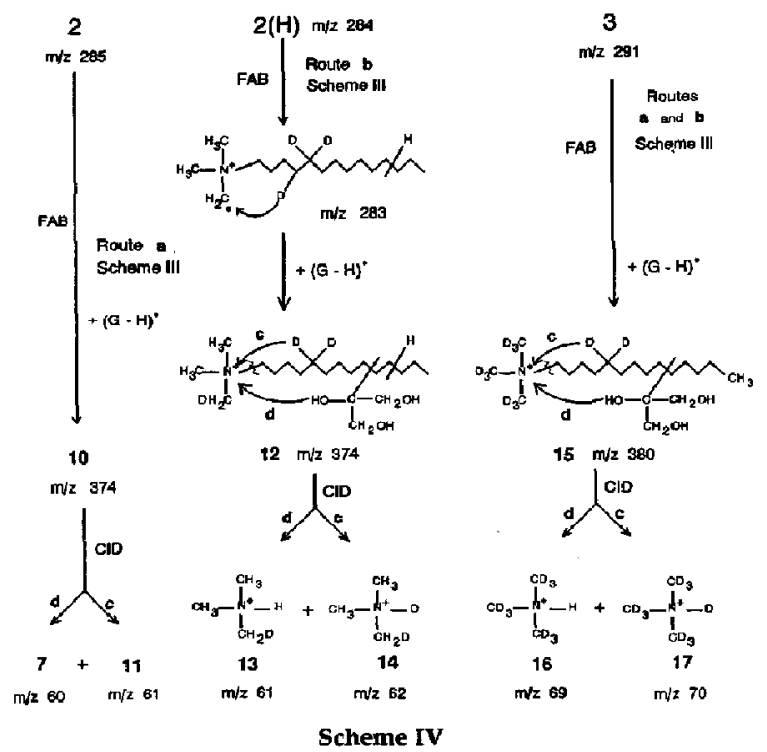


mation from $\mathbf{3}$ by either route $\mathbf{a}$ or $\mathbf{b}$ (analogous to Scheme III) leads to $15(m / z 380)$, because the $N$ methyl groups of 3 are already perdeuterated. As a consequence, the CID of $m / z 380$ derived from 3 generates fragments of $m / z 69$ and 70 in a ratio dictated by the reaction cross-sections for hydrogen versus deuterium migration. Cation 3 should not generate any fragment at $m / z 71$.

The pertinent time dependencies are depicted in Figure 4. Traces $E, F$, and $G$ of Figure $4 b$ render the observed intensities for $m / z 69,70$, and 71 from the reaction of 3. As expected, the abundance of $m / z 71$ (trace G) is negligible. ${ }^{\dagger}$ Thus, the data of Figure $4 \mathrm{~b}$ can be used to estimate the contribution from 11 to the signal at $m / z 61$ (designated $\mathrm{I}_{61(11)}$ ) by assuming that $I_{60(7)} / I_{61(11)}=I_{69(16)} / I_{70(17)}$ at each point in time. Because data in Figure 4 were acquired simultaneously from an equimolar mixture of 2 and 3 , identical experimental conditions are ensured. $\mathrm{I}_{61(13)}$ can either be estimated by difference $\left(I_{61(13)}=I_{61}-I_{61(11)}\right)$ or by a similar proportionality $\left(\mathrm{I}_{61(13)} / \mathrm{I}_{61(14)}=\mathrm{I}_{69(16)} / \mathrm{I}_{70(17)}\right)$. These two approaches yield the same result within $3 \%$. $I_{61(11)} / I_{62(14)}$ or $I_{64(7)} / I_{61(13)}$ represents the ratio of intermediate products 10 and 12 formed by the condensation reactions of 2 and $2(\mathrm{H})$. When adjusted for the known ratio of 2:2(H), it follows that routes $\mathbf{a}$ and $b$ of Scheme III compete in the ratio of approximately 4.5:1. While the precision of this result is not high, clearly the intramolecular radical transfer reaction is not negligible and should be taken into account when interpreting $\mathrm{FAB}$ or liquid secondary ion mass spectra that generate bombardment-damage products via radical mechanisms.

The very small signal observed for $m / z \quad 71$ is derived from tail attack on doubly mislabeled 3 (containing an extra $\mathbf{H}$ in the tail and a ${ }^{13} \mathrm{C}$ in the head to produce an adduct of $\mathrm{m} / \mathrm{z} 380$, which undergoes CID via routes $c$ and $\mathbf{d}$, Scheme IV. Route $\mathbf{d}$ produces $m / z$ 71. The small intensity of this precursor accounts for the small (negligible) intrensity at $\mathrm{m} / \mathrm{z} 71$.

10
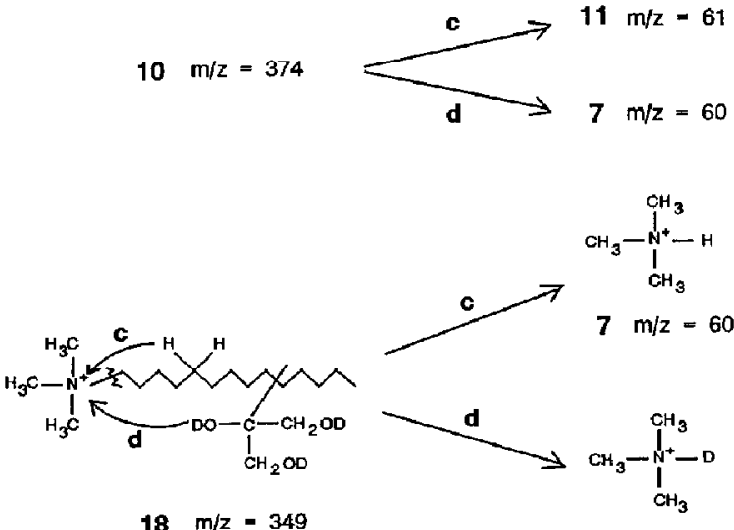

$11 \mathrm{~m} / \mathrm{z}=61$
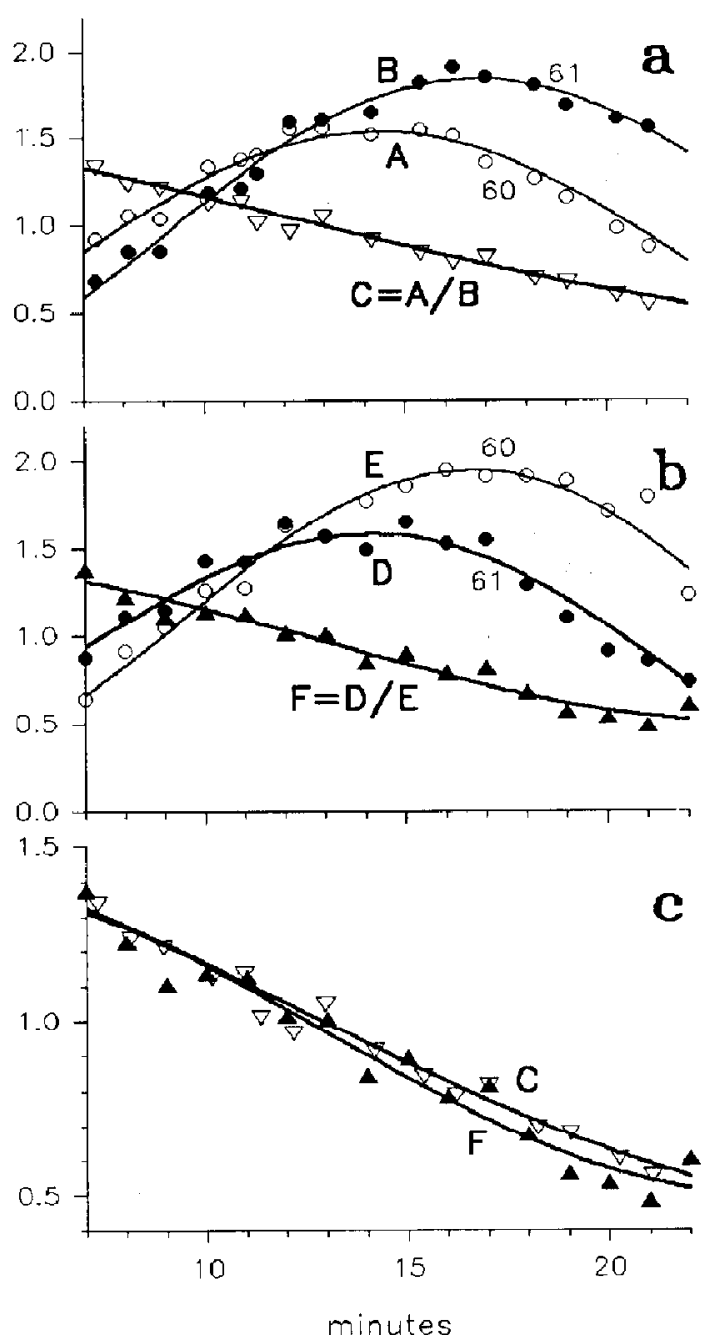

Figure 5. Time-dependent abundances of the CID fragment ions at the indicated mass-to-charge ratios. (a) Precursor ion is $\mathrm{m} / \mathrm{z}$ 374 , generated in situ by FAB of 2 in glycerol (Scheme V). (b) Precursor ion is $m / z \quad 349$, generated in situ by FAB of 1 in $\mathrm{d}_{3}$-glycerol (Scheme V). (c) Abundance ratios from parts $a$ and $b$ are compared.

\section{Kinetic Isotope Effect}

The CID of 10 to produce the fragments 7 and 11 (Scheme II) is potentially subject to a kinetic isotope effect. To probe the magnitude of this effect (if any), experiments were conducted using the nondeuterated surfactant $\mathbf{1}$ in conjunction with trideutero-glycerol $\left(d_{3} G\right)$, and the selectively deuterated surfactant 2 in conjunction with normal glycerol (G). Scheme $V$ incorporates the relevant reactions from Scheme IV and adds the reactions for formation of $\left(1+d_{3} G \rightarrow\right) 18 \rightarrow$ $7+11$. In each case the CID route $c$ represents transfer of a deuterium (hydrogen) from the hydrocarbon chain of the surfactant, and route $d$ represents the transfer of a hydrogen (deuterium) from a hydroxy-group of the 
glyceryl moiety (which is preferred, relative to the glyceryl C-H hydrogens [24]). For 10 the higher mass ion $(m / z$ 61) derives from route $c$, and for 18 the higher mass ion derives from route $d$. Any discernible kinetic isotope effect will become evident as a difference in the abundance ratios $I_{60} / I_{61}$ derived from CID of 10 and $I_{61} / I_{60}$ derived from CID of 18. Figure 5 shows the CID results for precursor ions 10 and $18 .^{*}$ For direct comparison, the two ratio curves are brought together in Figure 5c. Within the limits of experimental accuracy there is no difference in the intensity ratios. Therefore, there appears to be no significant kinetic isotope effect operative in the CID reaction.

\section{Conclusions}

The understanding of time-dependent phenomena in FAB and LSIMS may, in certain cases, be greatly enhanced by extending the studies to include the time dependence of tandem mass spectra. This has proven to be a powerful tool in explaining the mechanisms leading to bombardment-induced artifacts in the FAB spectra of trimethylammonium surfactants. Results with this system emphasize the sensitivity of the technique to even subtle structural variations of the starting materials and the reaction products.

\section{Acknowledgments}

This work was supported in part by a grant from the National Science Foundation (CHE-88-22787). The UTK Chemistry Mass Spectrometry Center is funded by the Srience Alliance, a State of Tennessee Center of Excellence. The NSF Chemical Instrumentation Program also contributed to the acquisition of the ZAB-EQ (grant CHE-86-09251).

\footnotetext{
${ }^{\ddagger}$ The perturbation caused by reaction $12 \rightarrow 13$ (Scheme IV) has been considered and can be shown to be negligible within the limits of the experimental accuracy of the data in Figure 5.
}

\section{References}

1. Detter, L. D.; Hand, O. W.; Cooks, R. G.; Walton, R. A. Mass Spectrom. Rev. 1988, 7, 465-502.

2. Paul, G. J.; Bourg, S.; Bertrand, M. J. I. Am. Soc. Mass Spectrom. 1993, 4, 493-503, and references therein.

3. Caldwell, K. A.; Gross, M. L. I. Am. Soc. Mass Spectrom. 1994, $5,73-92$.

4. Sunner, J. J. Am. Soc. Mass Spectrom. 1993, 4, 410-418.

5. Agnello, A.; De Pauw, E; Natalis, P. J. Am. Soc. Mass Spectrom. 1993, 4, 312-321.

6. Santana-Marques, M. G. O.; Ferrer-Correia, A. J. V.; Caldwell, K. A.; Gross, M. L. I. Am. Soc. Mass Spectrom. 1993, 4, 819-827.

7. Tuinman, A. A.; Cook, K. D. J. Am. Soc. Mass Spectromt. 1992, $3,318-325$.

8. Field, F. H. I. Phys. Chem. 1982, 86, 5115-5123.

9. Cole, R. B.; Guenat, C.; Hass, J. R.; Linton, R. W. Anal. Chem. 1987, 59, 1930-1937.

10. Gale, P. J.; Bentz, B. L.; Chait, B. T.; Field, F. H.; Cotter, R. J. Anat. Chem. 1986, 58, 1070-1076.

11. Wong, S. S.; Röllgen, F. W. Nucl. Inst. Methads 1986, B14, 436-447.

12. Todd, P. J.; Groenewold, G. S. Anal. Chem. 1986, 58, 895-899.

13. Ligon, W. V.; Dorn, S. B. Int. J. Mass Spectrom. Ion Processes 1984, 57, 75-90.

14. Ligon, W. V.; Dorn, S. B. Int. J. Mass Spectrom. Ion Processes 1986, 78, 99-113.

15. Lacey, M. P.; Keough, T. Rapid Comm. Mass Spectrom. 1989, 3, 46-50.

16. Kriger, M. S.; Cook, K. D.; Short, R. T.; Todd, P. J. Anal. Chem. 1992, 64, 3052-3058.

17. Scott, A. B.; Tartar, H. V. J. Am. Chem. Soc. 1943, 65, 692-698.

18. Tuinman, A. A,; Cook, K. D.; Magid, L. J. J. Am. Soc. Mass Spectrom. 1990, 1, 85-91.

19. Myers, D. Surfactant Science and Technology; VCH Publishers: New York, 1988; p 204.

20. Visentini, J.; Zidarov, D.; Allard, M.; Bertrand, M. J. J. Am Soc. Mass Spectrom. 1993, 4, 482-492.

21. Tsang, W. J. Am. Chem. SoC. 1985, 107, 2872-2880.

22. Castelhano, A. L.; Griller, D. J. Am. Chem, Soc. 1982, 104, 3655-3659.

23. Huang, R. L.; Goh, S. H.; Ong, S. H. The Chemistry of Free Radicals; Edward Amold: London, 1974; p 108.

24. Audier, H. E.; Milliet, A.; Perret, C.; Tabet, J. C.; Varenne, P. Nouveau J. Chim. 1977, 1, 269-270. 